\title{
miR-597 inhibits breast cancer cell proliferation, migration and invasion through FOSL2
}

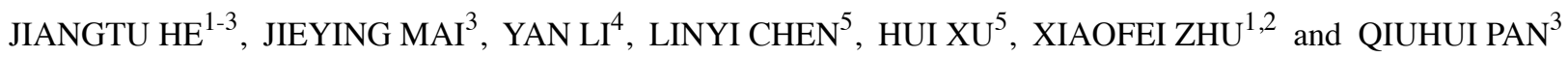 \\ ${ }^{1}$ The Research Center for Immunology, School of Laboratory Medicine, and ${ }^{2}$ Collaborative Innovation \\ Center of Molecular Diagnosis and Laboratory Medicine, Xinxiang Medical University, Xinxiang, Henan 453003; \\ ${ }^{3}$ Department of Central Laboratory, Tenth People's Hospital of Tongji University, Shanghai 200072; \\ ${ }^{4}$ Department of Clinical Laboratory, Maternal and Child Health Care of Zaozhuang, Zaozhuang, Shandong 277102; \\ ${ }^{5}$ Department of Ophthalmology, Tai Zhou Hospital of Zhejiang Province, Taizhou, Zhejiang 317099, P.R China
}

Received August 1, 2016; Accepted December 21, 2016

DOI: $10.3892 /$ or.2017.5558

\begin{abstract}
Many reports suggest that the discovery of microRNAs (miRNAs) might provide a novel therapeutical target for many diseases, even of human cancers; however, there are no reports on the role of miR-597 in human cancers. In the present study, by detecting mRNA expression with qRT-PCR, compared with the adjacent normal tissues we found that miR-597 was significantly downregulated in breast cancer tissues. By using the MTT assay, the cell woundhealing assay and the cell invasion assay, we demonstrated that miR-597 mimics were able to suppress breast cancer cell proliferation, migration and invasion. Additionally, with flow cytometry, we found that miR-597 influenced the growth of breast cancer cells through regulating the G1-S phase transition. Furthermore, we identified one binding site for miR-597 at the 3'UTR of the FOSL2 gene, using bioinformatics methods and the luciferase reporter assay, it was confirmed that FOSL2 was a direct target of miR-597. Moreover, overexpression of FOSL2 in MDA-MB-231 and SK-BR-3 cells can block the vast majority of the miR-597 roles, suggesting that miR-597 acts as a tumor suppressor in breast cancer cells by the downregulation of FOSL2. Additionally, we also found a negative correlation between the expression of FOSL2 and miR-597 in the tumor samples. This new regulatory mechanism in breast cancer may provide another method for diagnosis and therapy.
\end{abstract}

Correspondence to: Professor Qiuhui Pan, Department of Central Laboratory, Tenth People's Hospital of Tongji University, No. 301 Middle Yanchang Road, Shanghai 200072, P.R. China

E-mail: qiuhui_pan@126.com

Professor Xiaofei Zhu, The Research Center for Immunology, School of Laboratory Medicine, Xinxiang Medical University, No. 601 Jinsui Road, Xinxiang, Henan 453003, P.R. China

E-mail: zhu_xiaofei@126.com

Key words: miR-597, cell proliferation, migration, invasion, FOSL2

\section{Introduction}

Breast cancer, with a high mortality around the world, is one of the most frequent cancers among women. There are more than 1.7 million new cases of breast cancer worldwide in one year, with more than 522,000 deaths (1). Despite the fact that both basic scientists and clinical researchers have carried out many investigations on breast cancer, the prognosis remains unfavorable, the main reasons are attributed to the immature diagnosis and therapy and the adverse effects of surgeries $(2,3)$.

MicroRNAs (miRNAs) are short non-coding RNA molecules which are 20-25-nt long highly conserved and bind primarily to sequences within the $3^{\prime}$ untranslated region (3'UTR) of mRNAs. The primary function of miRNAs is to regulate its target gene expression by degradation or posttranscriptional regulation $(4,5)$. miRNAs target mRNA with a semi-complimentary seed sequence (6-9 bp) which guides binding to the response elements. One miRNA may have many potential targets because each seed sequence may match many mRNA molecules (5). Hundreds of miRNAs are found in well-defined transcriptional units, and can be in either intronic or exonic regions in non-coding transcriptional regions, or as intronic miRNAs in coding regions (6). Several previous studies have proposed that microRNAs are involved in a broad range of biological processes through their regulation of complex signal transduction pathways $(6,7)$. Half of human miRNAs can lead to the dysregulation of oncogenes and tumor suppressors in cancers by locating at fragile sites (8). Recent studies have revealed that miRNA signatures can stratify molecular subgroups of breast cancer $(9,10)$. Moreover, some miRNAs are able to predict the tumor progression and there are some reports on prognosis of patients with breast cancer $(11,12)$. There is only one study on miR-597 in pediatric patients with immune thrombocytopenic purpura (13), but there have been no studies on the function of miR-597 in breast cancer.

Fos-related antigen 2 (FOSL2), also named FRA-2, belongs to the AP-1 transcription factor family which includes the various isoforms of Fos and Jun (14). FOSL2 has been reported as a specific function gene in bone development (15) and appears to associate with diverse physiological and pathological processes, such as photoperiodic regulation (16) 
fibrosis (17) and even in cancer (18). In addition, FOSL2 has been documented as a key determinant of cellular plasticity during CD4 T cell differentiation (19). Some studies have reported that FOSL2 plays a key role in the regulation in some pathways, such as TGF- $\beta$ pathway, for example, FOSL 2 induce TGF- $\beta$ expression as a transcriptional regulator in cardiac fibroblasts (20). In human breast cancer, FOSL2 expression has been reported to enhance invasive properties $(20,21)$, but there have been few studies on the other functions.

In the present study, we first found that miR-597 was significantly downregulated in breast cancer tissues compared with the adjacent normal tissues. We then studied the expression and function of miR-597 in breast cancer cells. We also verified that FOSL2 was a direct target of miR-597, and that miR-597 negatively regulated FOSL2 expression to inhibit cell proliferation, migration and invasion in breast cancer.

\section{Materials and methods}

Cell culture and clinical specimens. Five breast carcinoma cell lines (T-47D, SK-BR-3, MDA-MB-231, MCF7 and BT-474) were purchased from the Chinese Academy of Science Cell Bank (Shanghai, China). Esophageal squamous cell carcinoma (EC-109), human cervical adenocarcinoma (HeLa), prostate cancer (PC1) and MCF10A cells were kindly provided by the Central Laboratory, Tongji University Affiliated People's 10th Hospital (Shanghai, China). All cells except MCF10A were cultured with $10 \%$ fetal bovine serum (FBS; Gibco-BRL/Life Technologies, Paisley, UK) in high-glucose Dulbecco's modified Eagle's medium (DMEM; HyClone Laboratories, Inc., Logan, UT, USA) and supplemented with antibiotics (100 U/ml penicillin and $100 \mathrm{mg} / \mathrm{ml}$ streptomycin; Gibco-BRL, Grand Island, NY, USA) at $37^{\circ} \mathrm{C}$ with $5 \% \mathrm{CO}_{2}$ in a humidified incubator. The MCF10A was maintained in complete media [DMEM/F12 (50:50 mix) supplemented with 5\% horse serum, $10 \mathrm{mg} / \mathrm{ml} 10 \mathrm{mM}$ HEPES, insulin, $20 \mathrm{ng} / \mathrm{ml}$ epidermal growth factor, $0.5 \mathrm{mg} / \mathrm{ml}$ hydrocortisone and $100 \mathrm{ng} / \mathrm{ml}$ cholera toxin]. Thirty-eight pair-matched breast tissue samples were obtained from the Department of Breast and Thyroid Surgery, Tongji University Affiliated People's 10th Hospital (Shanghai, China). All samples were obtained surgically and immediately snapfrozen and stored in liquid nitrogen until use. The Institutional Review Board approved the tissue procurement protocol used in the study, and the appropriate informed consent was obtained from the patients.

RNA isolation and quantitative real-time PCR. Total RNA was isolated by TRIzol reagent according to the manufacturer's protocol (Invitrogen, Carlsbad, CA, USA). cDNA was performed using $1000 \mathrm{ng}$ of total RNA as the template with looped primers and using a PrimeScript RT reagent kit (RR037A; Takara Bio, Inc., Shiga, Japan). Quantitative realtime PCR was performed in a 96-well plate according to the protocol of the KAPA SYBR FAST Universal qPCR kit (KK4601; Kapa Biosystems, Wilmington, MA, USA). An ABI 7900HT PCR sequencer (Applied Biosystems, Foster City, CA, USA) was used for the data measured, and gene expression was calculated using the $\Delta \Delta \mathrm{Ct}$ method for relative quantitation using $18 \mathrm{~S}$ or U6 as endogenous reference genes. The sequences of specific primers are 5'-GAGAGGAACA
AGCTGGCTGC-3' and 5'-GCTTCTCCTTCTCCTTCTGC-3' for FOSL2; 5'-CGTACAGCATCCTGGAGATA-3' and 5'-CC TTCAGCTTACAGTCATTG-3' for FGF10; 5'-CTGAGCTC TCTGAGTGCAAC-3' and 5'-GGTCTGAGCTGTATCGC TGC-3' for EGFR; 5'-GCAAGACTCCAGCGCCTTCT-3' and 5'-CTCATCTTCTTGTTCCTCCTC-3' for $M Y C$; 5'-CCTGG ATACCGCAGCTAGGA-3' and 5'-GCGGCGCAATACGAA TGCCCC-3' for 18S RNA; 5'-ACACTCCAGCTGGGTGTG TCACTCGATGAC-3' and 5'-TGGTGTCGTGGAGTCG-3' for miR-597; 5'-CTCGCTTCGGCAGCACA-3' and 5'-AACGCT TCACGAATTTGCGT-3' for $U 6$.

Cell proliferation, migration and invasion assays. Cell proliferation was detected using MTT assay, 1,000 cells transfected with synthesized oligonucleotides were seeded in 96-well plates. At 0, 48, 72 and $96 \mathrm{~h}, 100 \mu \mathrm{l}$ of 3-(4,5-dimethylthiazol-2-yl)-2,5-diphenyltetrazolium bromide (MTT) solution $(0.5 \mathrm{mg} / \mathrm{ml})$ (Sigma-Aldrich, St. Louis, MO, USA) was added to each well, after incubated for another $4 \mathrm{~h}$ the medium was removed, and $150 \mu \mathrm{l}$ of dimethyl sulfoxide (DMSO) was added. The plates were read at a wavelength of $490 \mathrm{~nm}$. Cell migration was detected by the wound-healing assay, an artificial wound was created by a $200 \mu$ l pipette tip. To visualize migrated cells and wound healing, images were taken at 0 and $36 \mathrm{~h}$. Cell invasion was detected using a Transwell chamber (Millipore, Billerica, MA, USA) with Matrigel (BD Biosciences, San Jose, CA, USA). Cells $\left(1 \times 10^{5}\right)$ were cultured in the top chamber of Transwell coated with Matrigel. Cells were cultured in medium with no fetal bovine serum (FBS) in the upper chamber and added with FBS-DMEM in the lower chamber. After $36 \mathrm{~h}$, invasive cells on the lower chamber were fixed in $95 \%$ ethanol and stained with $0.5 \%$ crystal violet, the numbers were determined using ImageJ software (National Institutes of Health, Bethesda, MD, USA).

Cell cycle assay. A total of $5 \times 10^{5}$ transfected cells were seeded into 6-well plates and incubated in $0.2 \%$ serum medium for cell synchronization. Subsequently, the cells were incubated with complete medium for $48 \mathrm{~h}$. The cells were stained with propidium iodide (PI) (550825; BD Biosciences, San Diego, CA, USA) and $\sim 1 \times 10^{5}$ cells were examined with a FACS flow cytometer and analyzed with ModFit software (BD Biosciences, San Jose, CA, USA).

EdU assay. A total of $1.5 \times 10^{5}$ transfected cells were plated into 6-well plates, and the assay performed according to the manufacturer's protocol of a Cell-Light ${ }^{\mathrm{TM}}$ EdU Apollo ${ }^{\circledR} 488$ In Vitro Imaging kit (C10310-3; Guangzhou RiboBio, Co., Ltd., Guangzhou, China). Finally, cells were visualized and counted using a fluorescence microscope (Leica Microsystems, Wetzlar, German).

Luciferase reporter assays. We used the pGL3-reporter luciferase vector to construct the pGL3-FOSL2 3'UTR or pGL3-FOSL2 3'UTR-mut vectors. The pGL3-FOSL2 3'UTRmut vector was obtained using a KOD-Plus-Mutagenesis kit (F0936K; Toyobo, Co., Ltd., Osaka, Japan). The Luciferase activity of NC and miR-597 were measured whit the Dualluciferase reporter assay system (Promega Corp., Fitchburg, WI, USA) after transfection for $36 \mathrm{~h}$. Firefly luciferase activity 
A
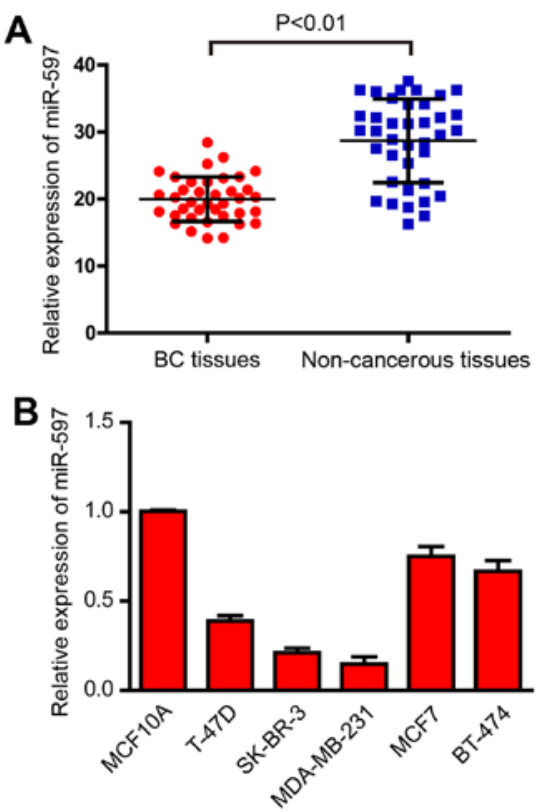

Figure 1. Expression of miR-597 in breast cancer tissues and cell lines. (A) miR-597 expression levels calculated by the $2^{-\mathrm{ACt}}$ method and normalized to U6 small nuclear RNA was detected by qRT-PCR in 38 tumor samples and pair-matched adjacent non-cancerous tissues. $P<0.01$. (B) miR-597 expression levels was detected by qRT-PCR in breast cancer cell lines T-47D, SK-BR-3, MDA-MB-231, MCF7, BT-474 and normal breast cell line MCF10A.

was normalized to Renilla luciferase activity for each transfected well. The sequences of specific primers are 5'-AT ACTCGAGAGCACCTTCAAGCGCTCCAG-3' and 5'-GG AAGCTTCTGCTGCTTGGATTCATCTC-3' for FOSL23'UTR, 5'-ATACTCGAGATGTACCAGGATTATCCCGG-3' and 5'-GCGGAATTCTTACAGAGCCAGCAGAGTGG-3' for FOSL2-CDS.

Western blot analysis. Protein lysates were separated by sodium dodecyl sulfate (SDS)-polyacrylamide gel electrophoresis and transferred to nitrocellulose membranes (Millipore, Billercia, MA, USA). The primary antibodies were incubated overnight after blocking with 5\% fat-free milk for $1 \mathrm{~h}$ and the primary antibodies were $\beta$-actin (Santa Cruz Biotechnology, Inc., Santa Cruz, CA, USA), FOSL2 (ab124830; Abcam, Cambridge, UK), MMP-2 and MMP-9 (13132, 13667; Cell Signaling Technologies, Beverly, MA, USA) and then incubated with their corresponding secondary antibodies (LI-COR Biosciences, Lincoln, NE, USA). Odyssey LI-CDR scanner (BD Biosciences) were used for the membranes.

Statistical analysis. All statistical analyses were performed using the SPSS version 20.0 (SPSS, Inc., Chicago, IL, USA). The data are presented as the mean \pm SD. The statistical significance is shown as $\mathrm{P}<0.05, \mathrm{P}<0.01$.

\section{Results}

miR-597 is downregulated in breast cancer tissues and cells. In order to ascertain the expression of miR-597 in breast cancer, qRT-PCR was performed in 38 pairs of breast cancer and non-cancerous tissues. As shown in Fig. 1A, the
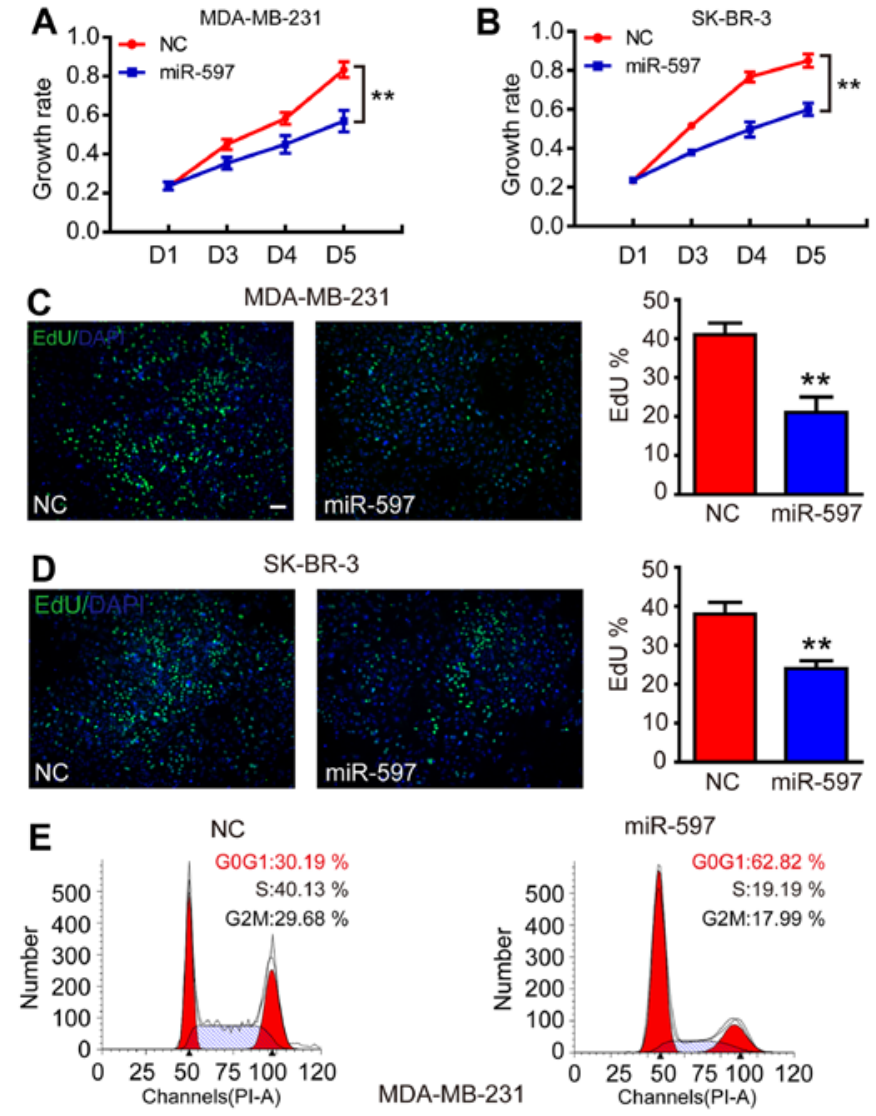

$\operatorname{miR}-597$
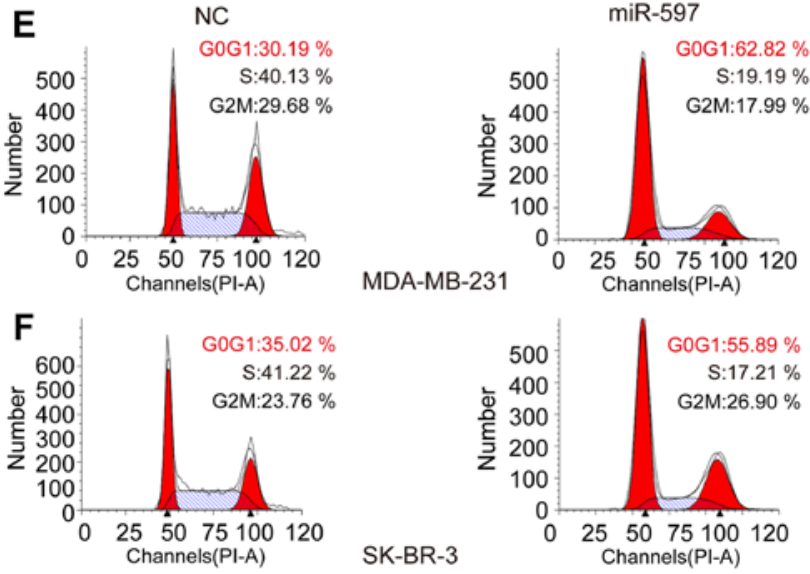

MDA-MB-231

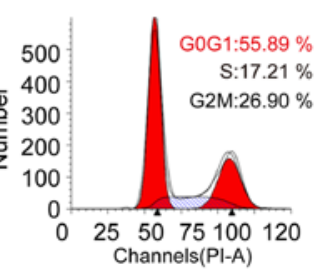

Figure 2. Effects of miR-597 mimic transfection on the proliferation of MDA-MB-231 and SK-BR-3 cells. (A and B) The growth rate was inhibited by the overexpression of FOSL2 in MDA-MB-231 and SK-BR-3 cells. The data were obtained using the MTT assay at $0,48,72$ and $96 \mathrm{~h}$ after transfection. (C and D) miR-597 inhibited the proliferation by impeding the entry of cells into S-phase. The nuclei of cells in S phase were stained with EdU (green), and the others were stained with DAPI (blue); bar, $20 \mu \mathrm{m}$. (E and F) miR-597 resulted in more G0/G1-phase cells. The number of cells in different phases was measured by flow cytometry with PI staining, ${ }^{* *} \mathrm{P}<0.01$.

results demonstrated that miR-597 was significantly downregulated in breast cancer tissues (Fig. 1A). Human breast cancer cell lines T-47D, SK-BR-3, MDA-MB-231, MCF7 and BT-474, and normal breast cell line MCF10A were also detected by qRT-PCR. As shown in Fig. 1B, the expression levels of miR-597 were also downregulated in the five breast cancer cell lines (Fig. 1B). Since MDA-MB-231 and SK-BR-3 cells exhibited the lowest miR-597 expression among the five breast cancer cell lines, these two cell lines were chosen for mature miR-597 mimic transfection and for further studies.

Overexpression of miR-597 inhibits cell proliferation. Subsequently, to evaluate the function of miR-597 in tumorigenesis, we transfected miR-597 mimics into MDA-MB-231 and SK-BR-3 to increase the levels of ectopic miR-597. As 
A

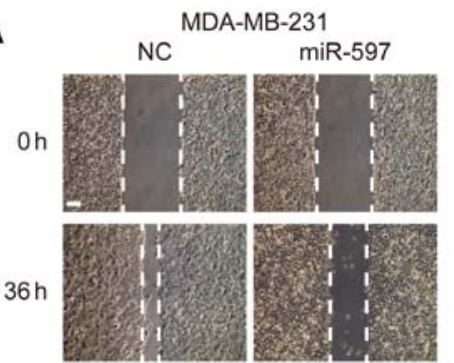

B

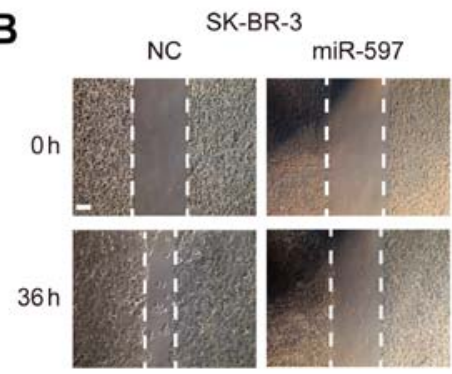

C

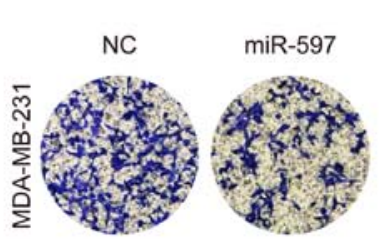

D

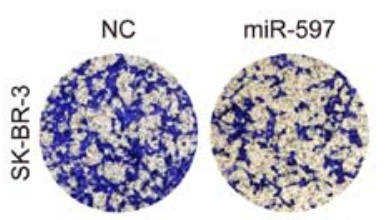

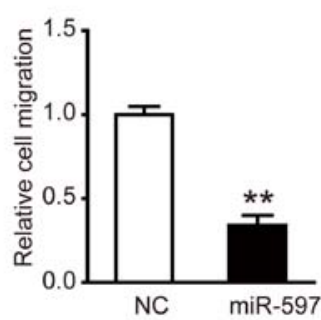
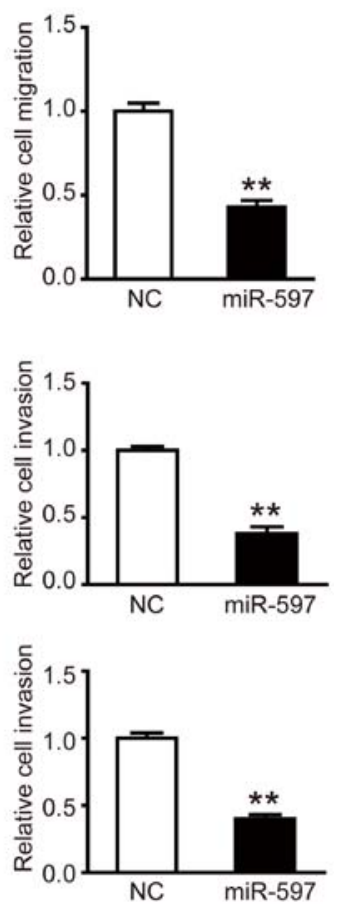

Figure 3. miR-597 inhibits the breast cancer cell migration and invasion. (A and B) Migration analysis of MDA-MB-231 and SK-BR-3 cells after treatment with NC and miR-597 mimics, relative ratio of wound closure per field is shown; bar, $100 \mu \mathrm{m}$. (C and D) Invasion analysis of MDA-MB-231 and SK-BR-3 cells after treatment with NC and miR-597 mimics; the relative ratio of invasive cells per field is shown, ${ }^{* *} \mathrm{P}<0.01$.

a result, the elevation of miR-597 significantly inhibited the growth rate of MDA-MB-231 and SK-BR-3 from the third day (Fig. 2A and $\mathrm{B} ; \mathrm{P}<0.01)$. The cell proliferation process was modulated by the cell cycle, thus, an EdU assay was performed. The result showed that the miR-597 subclone incorporated less EdU compared with the NC subclone (Fig. 2C and D; $\mathrm{P}<0.01$ ), indicating that miR-597 reduced cells into S-phase. In addition, flow cytometry revealed that cells with miR-597 overexpression had a $20-30 \%$ higher proportion at the G0/G1 phase and a $20 \%$ lower proportion at the $\mathrm{S}$ phase compared with NC groups (Fig. 2E and F). All results suggested that miR-597 inhibited cell proliferation by modulating the G1-S phase of the cell cycle.

Overexpression of miR-597 inhibits cell migration and invasion. To investigate the effect of miR-597 on the migration and invasion of breast cancer cells, miR-597 mimic or its negative control were transfected into MDA-MB-231 and SK-BR-3 cells, respectively. The wound healing assay was performed, the results showed that the migration ability of
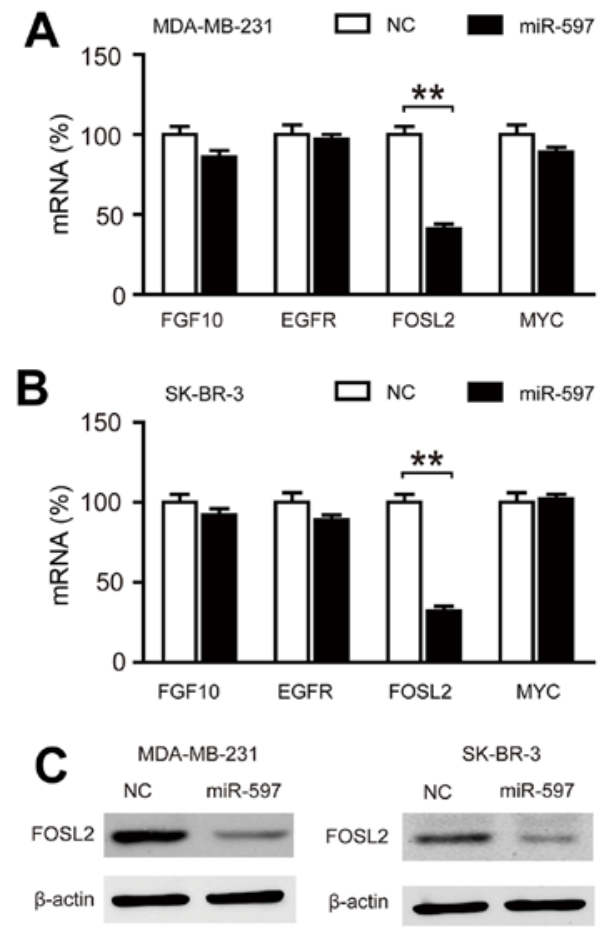
D FosL2 WT 3'UTR CCCGUGgagAAAGCAGUGACACA FOSL2 mUt 3'UTR CCCGUGGAGAAAGCACACUGUGA

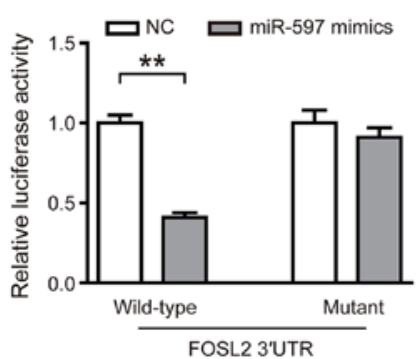

Figure 4. FOSL2 is the target of miR-597. (A and B) FOSL2 expression was inhibited by miR-597 at the mRNA level. Four genes (FGF10, EGFR, FOSL2 and MYC) that were chosen to examine the expression level between NC and miR-597 subclones using the $18 \mathrm{~S}$ ribosome as an internal reference. (C) miR-597 suppressed the expression of the FOSL2 protein. The expression level of FOSL2 was quantified by western blot analysis using $\beta$-actin as an internal reference. (D) Relative luciferase assay comparing the PGL3-FOSL2 and PGL3-FOSL2-mut vectors in MDA-MB-231 cells. Firefly luciferase activity was normalized to Renilla luciferase activity, ${ }^{* *} \mathrm{P}<0.01$.

MDA-MB-231 and SK-BR-3 cells in the miR-597 group was lower than $\mathrm{NC}$ groups at $36 \mathrm{~h}$ post-wounding (Fig. 3A and B; $\mathrm{P}<0.01)$. The cell invasion assay showed similar results, in the miR-597 group, there was $\sim 50 \%$ reduction in the average number of cells penetrating the Transwell membrane and Matrigel compared with NC group (Fig. $3 \mathrm{C}$ and $\mathrm{D} ; \mathrm{P}<0.01$ ). By detecting and analyzing with western blot analysis, we found that the expression levels of invasion-related proteins MMP-2 and MMP-9 were reduced (data not shown). Thus, the above results suggest that miR-597 plays an important part in the cell migration and invasion.

FOSL2 is a direct target of miR-597. In order to find the targets of miR-597, we used TargetScanHuman 6.2 and GeneCoDis3 

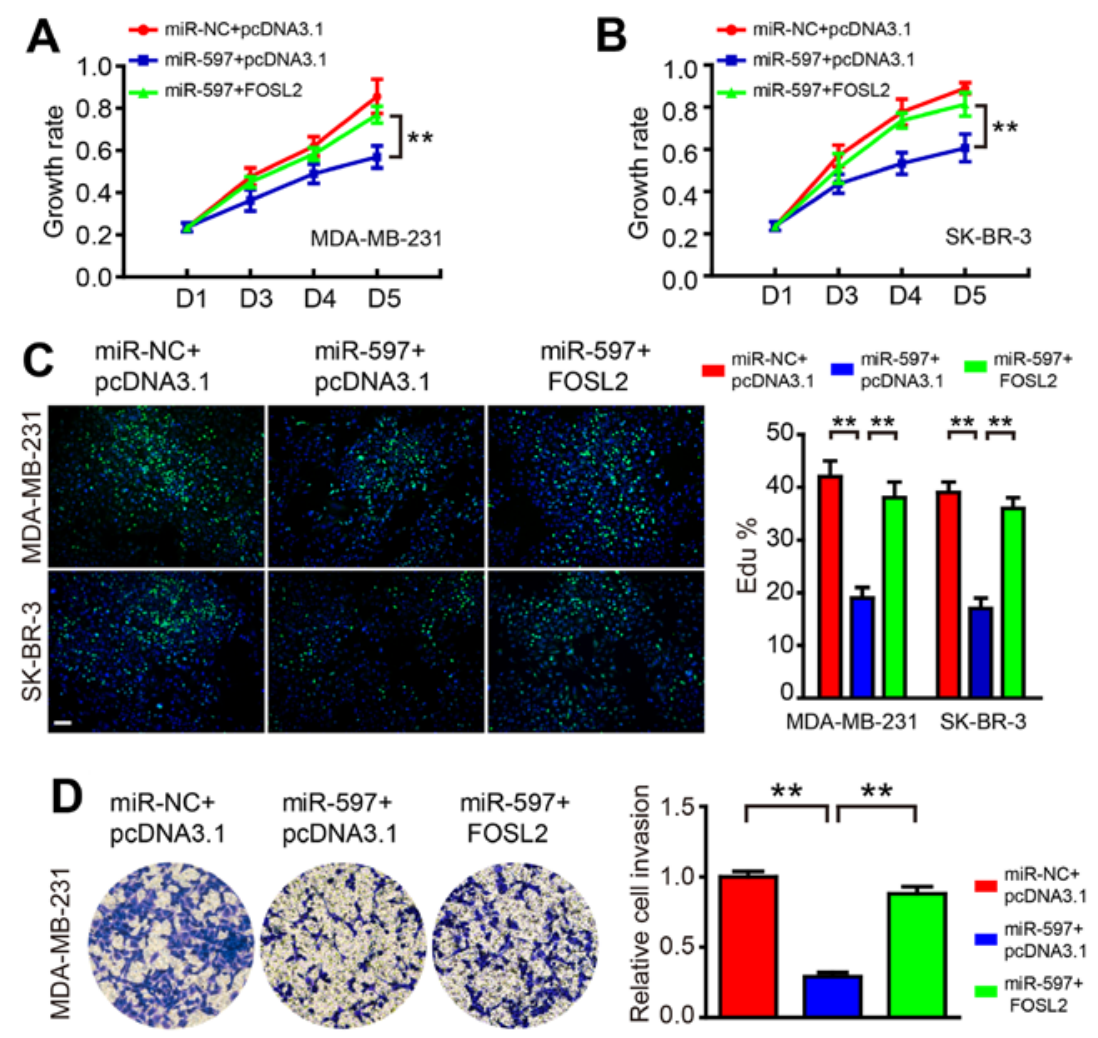

E
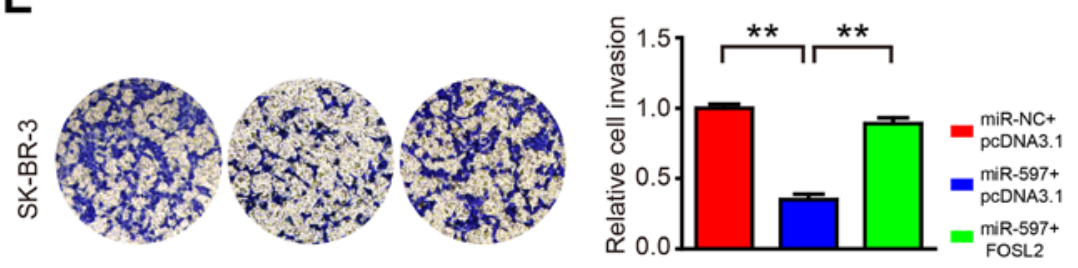

Figure 5. Overexpression of FOSL2 blocks the roles of miR-597. (A and B) The growth rate was promoted by the overexpression of FOSL2 in miR-597-overexpressing cells. The data were obtained using the MTT assay at $0,48,72$ and $96 \mathrm{~h}$ after transfection. (C) The overexpression of FOSL2 promoted proliferation by causing the entry of cells into S-phase. An EdU kit was used for the assay; bar, $20 \mu \mathrm{m}$. (D and E) The invasion rate was promoted by the overexpression of FOSL2 in miR-597-overexpression cells; the relative ratio of invasive cells per field is shown, ${ }^{* *} \mathrm{P}<0.01$.
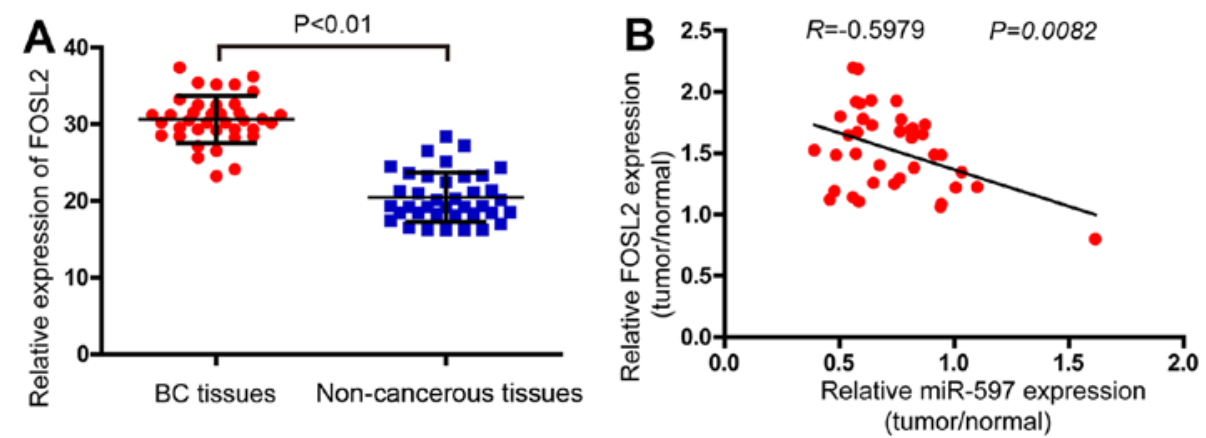

Figure 6. The correlations between FOSL2 and miR-597 in breast cancer tissues and normal breast tissues. (A) FOSL2 expression levels were detected by qRT-PCR in 38 tumor samples and pair-matched adjacent non-cancerous tissues using the $18 \mathrm{~S}$ ribosome as an internal reference; $\mathrm{P}<0.01$. (B) The negative correlation between the expression of miR-597 and FOSL2 in 38 patient samples with breast carcinoma $(r=-0.5979, \mathrm{P}=0.0082)$. The relative expression of miR-597 and FOSL2 was quantified using qRT-PCR, normalized to U6 or 18S and analyzed with the Pearson correlation. For comparisons, a two-tailed, unpaired t-test was used.

to predict the targets of miR-597 and we found that four genes (FGF10, EGFR, FOSL2 and MYC) had functions that oppose miR-597 in breast cancer. By performing qRT-PCR, we only found FOSL2 markedly inhibited by miR-597 (Fig. 4A and
$\mathrm{B} ; \mathrm{P}<0.01)$. In addition, the expression of FOSL2 protein in the miR-597 subclone was lower than that in the NC subclone (Fig. 4C). The luciferase reporter assays also demonstrated that miR-597 mimics significantly reduced the activity of 
FOSL2 3'-UTR, while there was no change in the mutant group (Fig. 4D). These data suggested that FOSL2 is a direct target of miR-597.

Overexpression of FOSL2 blocks the roles of $m i R-597$. To test the hypothesis that miR-597 regulates cell proliferation and invasion by targeting FOSL2, we co-transfected miR-597 mimics and FOSL2-expressing vector into MDA-MB-231 and SK-BR-3 cells. The western blot result showed that the level of FOSL2 decreased by miR-597 could be rescued by overexpression of FOSL2 in cells (data not shown). As shown in Fig. 5, FOSL2 reintroduction reversed the anti-proliferation and anti-invasion roles of miR-597, suggesting that the effect of miR-597 on cell growth and invasion were largely mediated by its downregulation of FOSL2.

Effect of FOSL2 and miR-597 on the clinicopathological features of breast cancer. In order to determine the expression levels of FOSL2 in breast cancer, the real-time PCR analysis was performed in 38 pairs of breast cancer and adjacent non-cancerous tissues. The results demonstrated that the FOSL2 mRNA were higher in tumor samples compared with the adjacent normal tissues (Fig. 6A). Finally, analyzing with Pearson correlation the expression of FOSL2 and miR-597 (Fig. 1A) in these 38 tumor samples, we found a negative correlation between the two (Fig. 6B, $R=-0.5979$; $\mathrm{P}=0.0082$ ).

\section{Discussion}

Increasing number of miRNAs are reported in different cancers. The altered expression of miRNAs lead to changes in cell proliferation, migration, invasion, survival and apoptosis underlying many diseases, included cancers. After the first report on deregulation of miRNA in human lymphocytic leukemia (22) many other studies showed aberrant expression profiles of miRNA in breast cancer. However, to the best of our knowledge, this is the first report on the expression level and biological function of miR-597 in breast cancer. In the present study, we did not only clearly demonstrate that miR-597 functioned as a tumor suppressor that inhibited breast cell proliferation, migration and invasion, but we also identified that FOSL2 was one of its target genes.

As the first investigation on miR-597 in breast cancer, we speculated whether miR-597 had similar influence on other types of cancers. Thus, the esophageal squamous cell line EC-109, the cervical cancer cell line HeLa and prostate cancer cell line PC1 were used for similar functional research (data not shown), and their proliferation was also inhibited by miR-597, the result suggested that miR-597 has similar function in regulating cell proliferation on other types of cancer cells. However, we detected the expression of FOLS2 in these types of cancers while overexpression of miR-597, we found that the prostate cancer cell line PC1 had an insignificant change of FOSL2 while others had a significant down-expression. We considered that the function of miR-597 in PC1 was not through FOSL2 but other targets, this finding suggested that FOSL2 is one of the targets of miR-597. There may be a number of other targets of miR-597 not yet found.
The AP-1 family is known to be involved in cellular proliferation and oncogenesis, depending on the combination of AP-1 proteins and the cellular context $(23,24)$. Fra-2 is one component of a functional AP-1 complex that can also comprise of Jun-Jun, Jun-Fos or Jun-ATF2 dimers (25). FOSL2 was originally identified as a gene encoding FOS related antigen gene-2 $(26,27)$ and shown to be oncogenic for chicken embryonic fibroblasts (CEFs) (27). In our studies, we found that FOSL2 was significantly upregulated in breast cancer tissues compared with the adjacent normal tissues. FOSL2 overexpression in the miR-597-overexpression cancer cells block the vast majority of miR-597 roles, which is consistent with previous studies $(20,21)$. FOSL2 may be one of the targets of miR-597 in breast cancer cells, it can partly rescue the effect in cell proliferation (Fig. 5A and B). Possibly there is another target of miR-597 on this function which should be studied further.

Various synthetic miRNAs have been reported to exert powerful tumor-suppressive effects in many different cancer types, such as bladder cancer, melanoma and gastric cancer (28-30), even in breast cancer, artificial miRNA which targeted CXCR4 or MTDH blocked the proliferation, invasion and metastasis $(31,32)$. All these findings suggested that artificially designed miRNA may be a valuable approach in the treatment of breast cancer. Although the synthetic miR-597 had powerful tumor-suppressive effects in breast cancer, the mechanism remains still unknown. Two main reasons were accepted for the abnormal versions of microRNAs: one is the promoter modification (28) and the other is the regulation of interacting proteins $(29,30)$. Regardless of the cause of the underexpression of miR-597, or which pathway was affected by miR-597further studied are required.

\section{Acknowledgements}

The authors wish to thank Jiayi Wang, Shanshan Xu, Ji Ma, Yue Zhang and Fenyong Sun of Shanghai Tongji University for their technical assistance. The present study was supported by the National Natural Science Foundation of China (grant no. 81371913).

\section{References}

1. Ferlay J, Soerjomataram I, Dikshit R, Eser S, Mathers C, Rebelo M, Parkin DM, Forman D and Bray F: Cancer incidence and mortality worldwide: sources, methods and major patterns in GLOBOCAN 2012. Int J Cancer 36: E359-E386, 2015.

2. Farazi TA, Hoell JI, Morozov P and Tuschl T: MicroRNAs in human cancer. Adv Exp Med Biol 774: 1-20, 2013.

3. Cuzick J, DeCensi A, Arun B, Brown PH, Castiglione M, Dunn B, Forbes JF, Glaus A, Howell A, von Minckwitz G, et al: Preventive therapy for breast cancer: A consensus statement. Lancet Oncol 12: 496-503, 2011.

4. Quintavalle M, Elia L, Condorelli G and Courtneidge SA: MicroRNA control of podosome formation in vascular smooth muscle cells in vivo and in vitro. J Cell Biol 189: 13-22, 2010.

5. Dang X, Ma A, Yang L, Hu H, Zhu B, Shang D, Chen T and Luo Y: MicroRNA-26a regulates tumorigenic properties of EZH2 in human lung carcinoma cells. Cancer Genet 205: 113-123, 2012.

6. Inui M, Martello G and Piccolo S: MicroRNA control of signal transduction. Nat Rev Mol Cell Biol 11: 252-263, 2010.

7. Cordes KR, Sheehy NT, White MP, Berry EC, Morton SU, Muth AN, Lee TH, Miano JM, Ivey KN and Srivastava D: miR-145 and miR-143 regulate smooth muscle cell fate and plasticity. Nature 460: 705-710, 2009. 
8. Xu X, Chen Z, Zhao X, Wang J, Ding D, Wang Z, Tan F, Tan X, Zhou F, Sun J, et al: MicroRNA-25 promotes cell migration and invasion in esophageal squamous cell carcinoma. Biochem Biophys Res Commun 421: 640-645, 2012.

9. Blenkiron C, Goldstein LD, Thorne NP, Spiteri I, Chin SF, Dunning MJ, Barbosa-Morais NL, Teschendorff AE, Green AR, Ellis IO, et al: MicroRNA expression profiling of human breast cancer identifies new markers of tumor subtype. Genome Biol 8: R214, 2007.

10. Lowery AJ, Miller N, Devaney A, McNeill RE, Davoren PA, Lemetre C, Benes V, Schmidt S, Blake J, Ball G, et al: MicroRNA signatures predict oestrogen receptor, progesterone receptor and HER2/neu receptor status in breast cancer. Breast Cancer Res 11: R27, 2009.

11. Foekens JA, Sieuwerts AM, Smid M, Look MP, de Weerd V, Boersma AW, Klijn JG, Wiemer EA and Martens JW: Four miRNAs associated with aggressiveness of lymph node-negative, estrogen receptor-positive human breast cancer. Proc Natl Acad Sci USA 105: 13021-13026, 2008.

12. Rothé F, Ignatiadis M, Chaboteaux C, Haibe-Kains B, Kheddoumi N, Majjaj S, Badran B, Fayyad-Kazan H, Desmedt C, Harris AL, et al: Global microRNA expression profiling identifies MiR-210 associated with tumor proliferation, invasion and poor clinical outcome in breast cancer. PLoS One 6: e20980, 2011.

13. Bay A, Coskun E, Oztuzcu S, Ergun S, Yilmaz F and Aktekin E: Plasma microRNA profiling of pediatric patients with immune thrombocytopenic purpura. Blood Coagul Fibrinolysis 25: 379-383, 2014.

14. Tulchinsky E: Fos family members: Regulation, structure and role in oncogenic transformation. Histol Histopathol 15: 921-928, 2000.

15. Bozec A, Bakiri L, Jimenez M, Rosen ED, Catalá-Lehnen $P$, Schinke T, Schett G, Amling M and Wagner EF: Osteoblastspecific expression of Fra-2/AP-1 controls adiponectin and osteocalcin expression and affects metabolism. J Cell Sci 126 : 5432-5440, 2013.

16. Engel L, Gupta BB, Lorenzkowski V, Heinrich B, Schwerdtle I, Gerhold S, Holthues H, Vollrath L and Spessert R: Fos-related antigen 2 (Fra-2) memorizes photoperiod in the rat pineal gland. Neuroscience 132: 511-518, 2005.

17. Roy S, Khanna S, Azad A, Schnitt R, He G, Weigert C, Ichijo H and Sen CK: Fra-2 mediates oxygen-sensitive induction of transforming growth factor beta in cardiac fibroblasts. Cardiovasc Res 87: 647-655, 2010 .

18. Zhou L, Graves M, MacDonald G, Cipollone J, Mueller CR and Roskelley CD: Microenvironmental regulation of BRCA1 gene expression by c-Jun and Fra2 in premalignant human ovarian surface epithelial cells. Mol Cancer Res 11: 272-281, 2013.

19. Ciofani M, Madar A, Galan C, Sellars M, Mace K, Pauli F, Agarwal A, Huang W, Parkhurst CN, Muratet M, et al: A validated regulatory network for Th17 cell specification. Cell 151: 289-303, 2012.
20. Schröder C, Schumacher U, Müller V, Wirtz RM, Streichert T, Richter U, Wicklein D and Milde-Langosch K: The transcription factor Fra-2 promotes mammary tumour progression by changing the adhesive properties of breast cancer cells. Eur J Cancer 46: 1650-1660, 2010.

21. Milde-Langosch K, Röder H, Andritzky B, Aslan B Hemminger G, Brinkmann A, Bamberger CM, Löning T and Bamberger AM: The role of the AP-1 transcription factors c-Fos, FosB, Fra-1 and Fra-2 in the invasion process of mammary carcinomas. Breast Cancer Res Treat 86: 139-152, 2004.

22. Calin GA, Dumitru CD, Shimizu M, Bichi R, Zupo S, Noch E, Aldler H, Rattan S, Keating M, Rai K, et al: Frequent deletions and down-regulation of micro- RNA genes miR15 and miR16 at $13 q 14$ in chronic lymphocytic leukemia. Proc Natl Acad Sci USA 99: 15524-15529, 2002.

23. Shaulian E and Karin M: AP-1 as a regulator of cell life and death. Nat Cell Biol 4: E131-E136, 2002.

24. Eferl R and Wagner EF: AP-1: A double-edged sword in tumorigenesis. Nat Rev Cancer 3: 859-868, 2003.

25. Daury L, Busson M, Tourkine N, Casas F, Cassar-Malek I, Wrutniak-Cabello C, Castellazzi M and Cabello G: Opposing functions of ATF2 and Fos-like transcription factors in c-Junmediated myogenin expression and terminal differentiation of avian myoblasts. Oncogene 20: 7998-8008, 2001.

26. Matsui M, Tokuhara M, Konuma Y, Nomura N and Ishizaki R: Isolation of human fos-related genes and their expression during monocyte-macrophage differentiation. Oncogene 5: 249-255, 1990.

27. Nishina H, Sato H, Suzuki T, Sato M and Iba H: Isolation and characterization of fra-2, an additional member of the fos gene family. Proc Natl Acad Sci USA 87: 3619-3623, 1990.

28. Li Z, Zhan W, Wang Z, Zhu B, He Y, Peng J, Cai S and Ma J: Inhibition of PRL-3 gene expression in gastric cancer cell line SGC7901 via microRNA suppressed reduces peritoneal metastasis. Biochem Biophys Res Commun 348: 229-237, 2006.

29. Liu Y, Han Y, Zhang H, Nie L, Jiang Z, Fa P, Gui Y and Cai Z: Synthetic miRNA-mowers targeting miR-183-96-182 cluster or miR-210 inhibit growth and migration and induce apoptosis in bladder cancer cells. PLoS One 7: e52280, 2012.

30. Liu X, Fang H, Chen H, Jiang X, Fang D, Wang Y and Zhu D: An artificial miRNA against HPSE suppresses melanoma invasion properties, correlating with a down-regulation of chemokines and MAPK phosphorylation. PLoS One 7: e38659, 2012.

31. Liang Z, Wu H, Reddy S, Zhu A, Wang S, Blevins D, Yoon Y, Zhang Y and Shim H: Blockade of invasion and metastasis of breast cancer cells via targeting CXCR4 with an artificial microRNA. Biochem Biophys Res Commun 363: 542-546, 2007.

32. Wang S, Shu JZ, Cai Y, Bao Z and Liang QM: Establishment and characterization of MTDH knockdown by artificial MicroRNA interference - functions as a potential tumor suppressor in breast cancer. Asian Pac J Cancer Prev 13: 2813-2818, 2012. 